\title{
BMJ Open Active surveillance for carbapenemase- producing Klebsiella pneumoniae and correlation with infection in subjects attending an Italian tertiary-care hospital: a 7-year retrospective study
}

To cite: Calderaro A, Buttrini M, Martinelli M, et al. Active surveillance for carbapenemase-producing Klebsiella pneumoniae and correlation with infection in subjects attending an Italian tertiary-care hospital: a 7-year retrospective study. BMJ Open 2021;11:e042290. doi:10.1136/ bmjopen-2020-042290

- Prepublication history for this paper is available online. To view these files, please visit the journal online (http://dx.doi org/10.1136/bmjopen-2020042290).

Received 01 July 2020 Revised 23 November 2020 Accepted 04 April 2021

Check for updates

(c) Author(s) (or their employer(s)) 2021. Re-use permitted under CC BY-NC. No commercial re-use. See rights and permissions. Published by BMJ.

${ }^{1}$ Department of Medicine and Surgery, University of Parma, Parma, Italy

${ }^{2}$ Unit of Clinical Microbiology, University Hospital of Parma, Parma, Italy

${ }^{3}$ Unit of Clinical Virology, University Hospital of Parma, Parma, Italy

Correspondence to Dr Adriana Calderaro; adriana.calderaro@unipr.it

\section{ABSTRACT}

Objectives The distribution of carbapenemase-producing Klebsiella pneumoniae (CPKP) phenotypes and genotypes in samples collected during 2011-2018 was evaluated. The association between patients with CPKP-positive rectal swab and those with CPKP infection, as well as the overall analysis of CPKP-infected patients, was performed. Setting The study was performed in a tertiary-care hospital located in Northern Italy.

Participants Two groups were considered: 22939 'atrisk' patients submitted to active surveillance for CPKP detection in rectal swabs/stools and 1094 CPKP-infected patients in which CPKP was detected in samples other than rectal swabs.

Results CPKP-positive rectal swabs were detected in $5 \%$ (1150/22 939). A CPKP infection was revealed in $3.1 \%$ (719/22 939) of patients: 582 with CPKP-positive rectal swab $(50.6 \%$ of the 1150 CPKP-positive rectal swabs) and 137 with CPKP-negative rectal swab. The $49.4 \%$ (568/1150) of the patients with CPKP-positive rectal swab were carriers. The overall frequency of CPKP-positive patients (carriers and infected) was almost constant from 2012 to 2016 (excluding the 2015 peak) and then increased in 2017-2018. blaKPC was predominant followed by blaVIM. No difference was observed in the frequency of CPKP-positive rectal swab patients among the different material groups. Among the targeted carbapenemase genes, blaVIM was more significantly detected from urine than from other samples.

Conclusions The high prevalence of carriers without evidence of infection, representing a potential reservoir of CPKP, suggests to maintain the guard about this problem, emphasising the importance of active surveillance for timely detection and separation of carriers, activation of contact precautions and antibiotic treatment guidance on suspicion of infection.

\section{INTRODUCTION}

Multidrug-resistant (MDR) bacteria represent an increasing public health threat in
Strengths and limitations of this study

- This study describes the distribution of the carbapenemase-producing Klebsiella pneumoniae (CPKP) phenotypes and genotypes detected in a large number of samples over a long period (7 years), with particular reference to their temporal trend.

- The results reported in this study from an unselected patient population attending a single tertiarycare hospital may contribute to the global data production.

- The association between patients with CPKPpositive rectal swab and those with CPKP infection was performed taking into account also the different material groups.

- The molecular genotypic characterisation based only on the four major carbapenemase genes could have missed the more rarely circulating genotypes.

- The lack of further genetic typing hampered to add consideration about any molecular epidemiological link among the isolates.

healthcare settings. Among MDR bacteria, carbapenemase-producing Enterobacteriaceae (CPE), especially Klebsiella pneumoniae, are a cause for concern being able to spread rapidly ${ }^{1}$ and responsible for invasive infections associated with high mortality, ${ }^{2}$ recently inducing the Centers for Disease Control and Prevention (CDC) to raise them to the highest threat level. ${ }^{3}$ The application of CPE control programmes has been successful in some areas; however, the problem continues to worsen worldwide, requiring more effective prevention strategies. ${ }^{14}$

Carbapenemases are enzymes included in the Amber classification in the A, B or D 
classes. The class A and D enzymes are serine hydrolases, and the class B or metallo-beta-lactamase (MBL) enzymes are catalases requiring one or two zinc ions on the active site. ${ }^{5}$ Although certain carbapenemases are typically associated with specific regions or countries, nowadays, due to globalisation especially in terms of widespread international travel and broad access to medical care, such an association may change, emphasising the need for routine local and national surveillance. ${ }^{4}$ In particular, in Italy, besides the detection of Verona integron-encoded metallo-beta-lactamase (VIM)-producing Enterobacteriaceae, first detected in the early 2000s, K. pneumoniae carbapenamase (KPC) producers are widely spread whereas New Delhi metallo-beta-lactamase (NDM) and carbapenem-hydrolysing oxacillinase-48 (OXA-48) producers are only occasionally revealed. ${ }^{3}$

The aim of this study was the evaluation of the distribution of the phenotypes and genotypes of the carbapenemase-producing K. pneumoniae (CPKP) strains circulating in two selected groups of patients (those examined for CPKP detection on rectal swab as part of the National/Regional active carbapenem-resistant Enterobacteriaceae (CRE) surveillance, and those with a CPKP infection) in a tertiary-care hospital during a 7-year period (2011-2018). The association between patients with CPKP-positive rectal swab and those with CPKP infection, as well as the overall analysis of CPKP-infected patients, was performed.

\section{METHODS}

\section{Study design}

The study was designed as a retrospective data collection. The total observation time was 7 years.

Data were sought retrospectively from the records produced by the diagnostic flow of the laboratory, as answer to a clinical suspicion or to active CRE surveillance. ${ }^{6}$

\section{Patient and public involvement}

Patients were not involved in the study.

\section{Definitions}

A patient was defined as carrier when only a CPKP-positive rectal swab was detected and as infected when a CPKPpositive sample other than rectal swab was found, in presence of signs and symptoms of infection, according to CDC criteria for specific types of infections. ${ }^{7}$

CPKP-positive samples other than rectal swab were grouped in blood (including blood, vascular catheter and cerebrospinal fluid), respiratory (including bronchial aspirate, bronchoalveolar lavage, sputum, pleural fluid, pharyngeal swab, nasopharyngeal aspirate and nasal swab), urine (including urine and urinary catheter), and other (including bile, peritoneal, ascitic and abdominal drainage fluids, pus, bioptic and prothesic materials, sperm, tongue, wound, cutaneous, vaginal and urethral swabs).

\section{Study setting and population}

Two well-defined groups of patients attending a tertiarycare hospital (University-Hospital of Parma, Italy) from November 2011 to October 2018 were selected. The first group included 22939 'at-risk' patients (median age 70 years, range from 1 day to 108 years), for a total of 32477 rectal swabs due to multiple sampling when required by CRE surveillance. ${ }^{6}$ The second group included 1094 CPKP-infected patients (median age 78 years, range from 20 days to 102 years), either involved or not involved in active CRE surveillance. In case of multiple CPKP-positive samples, only the first one of each patient was considered. Multiple CPKP-positive samples categorised in the same material group (blood, respiratory, urine and other) and belonging to the same infected patient were considered only once, as a unique sample.

\section{Inclusion and exclusion criteria}

Inclusion criteria: the first group included 'at-risk' patients examined on admission as part of the National/Regional active CRE surveillance for the detection of CPKP strains in rectal swabs/stools (hereafter referred as rectal swab) ${ }^{6}$ according to the following indications: (1) contacts of CPKP-positive patients; (2) patients admitted to transplant surgery, intensive care units or any other 'at-risk' unit such as long-term care units, oncology and haematology; (3) patients known to be infected/colonised, with the last CPKP positivity dating back to more than 90 days from the new admission; (4) patients coming from endemic countries, such as Israel, Greece, Pakistan and India; (5) patients transferred from acute care and neurological rehabilitation facilities; (6) patients coming from nursing homes for the elderly; (7) patients hospitalised in an acute care facility in the last 6 months.

The second group included patients with a CPKP infection in at least a sample other than rectal swab, either involved or not involved in active CRE surveillance.

Exclusion criteria: no exclusion criteria were adopted.

\section{Microbiological methods}

The rectal swabs were inoculated onto chromogenic agar (Brilliance CRE medium, Oxoid, Milan, Italy) and the blue colonies referring to presumptive CRE were subcultured on MacConkey agar with a carbapenem disk, as previously described. ${ }^{3}$ CPKP strains from clinical samples other than rectal swabs were isolated, as previously described. ${ }^{3}$ All K. pneumoniae strains were identified by MALDI-TOF MS and submitted to antimicrobial susceptibility testing (Gram-negative NMIC/ID88 or NMIC/ ID94 Combo Panels, Becton Dickinson, Sparks, MD, USA). When a carbapenem nonsusceptible $K$. pneumoniae strain was revealed, the carbapenemase production confirmation was performed by phenotypical analysis and genotypical characterisation. The phenotypical analysis, according to Regional guidelines, ${ }^{6}$ included the modified Hodge test in combination with the disk diffusion inhibition test (KPC+MBL Confirm ID Kit/ KPC, MBL and OXA-48 Confirm Kit, Rosco Diagnostica, Taastrup, 
(A)

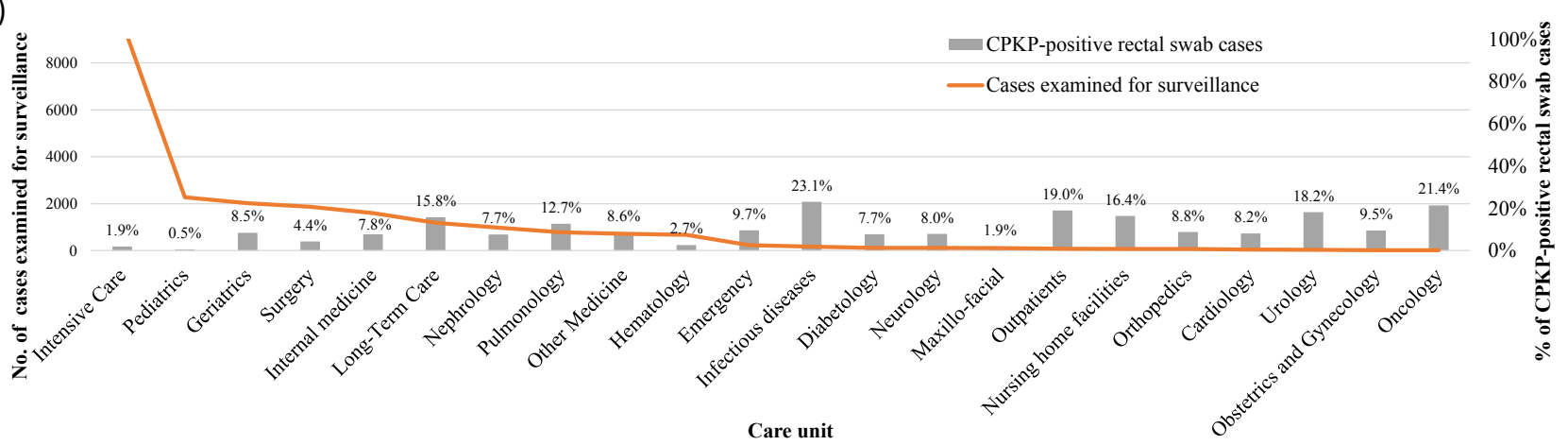

(B)

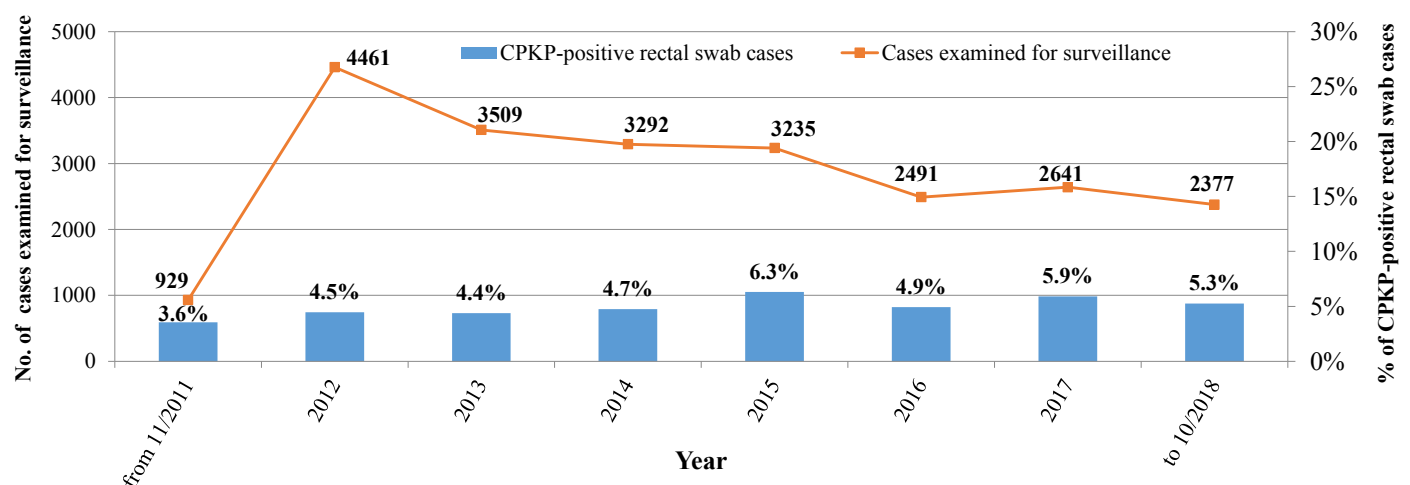

Figure 1 Distribution of carbapenemase-producing Klebsiella pneumoniae (CPKP)-positive rectal swab cases by care unit (A) and by year (B).

Denmark), able to differentiate KPC, MBL and OXA-48 like carbapenemases, performed according to the manufacturer's instructions. For the genotypical characterisation, two molecular methods were used during the study period: the first, detecting blaKPC, blaNDM, and retrospectively, on the available blaNDM-negative MBLproducing isolates, blaVIM, was used until 2015, and the second one also detecting blaOXA-48 was used since 2015 , as previously described. ${ }^{3}$

\section{Statistical analysis}

$\chi^{2}$ test was used for comparison of the frequency of CPKPpositive rectal swabs among CPKP-infected patients by material grouping, the frequency of involvement of multiple materials among CPKP-infected patients, and the distribution of carbapenemase genes in the different material groups. Statistical significance was set at $p<0.01$.

\section{RESULTS}

Among the 22939 'at-risk' patients (32477 rectal swabs), carbapenem-resistant $K$. pneumoniae strains were detected in 1178 cases $(5.1 \%)$ and the production of carbapenemase was revealed in 1150 cases (5\%). Intensive care and long-term care units accounted for the highest number of patients with CPKP-positive rectal swabs (188 cases each), with a prevalence of $1.9 \%$ and $15.8 \%$, respectively (figure 1A). The frequency of patients with CPKP-positive rectal swab ranged from $4.4 \%$ to $4.7 \%$ in the 2012-2014 period, reached the highest peak $(6.3 \%)$ in 2015 , and showed a fluctuating trend from 2016 to 2018 (figure 1B). With regard to the results of the molecular genotyping assays, all targeted types of carbapenemase genes were detected among the analysed rectal swabs. The blaKPC was predominant $(79 \%, 909 / 1150)$ followed by blaVIM $(16.7 \%, 192 / 1150)$, while blaOXA-48 and blaNDM were more rarely observed, accounting for $0.3 \%(3 / 1150)$ and $0.2 \%(2 / 1150)$, respectively (figure 2$)$. In $0.8 \%$ (9/1150, 8 class B other than blaNDM and blaVIM and 1 class A other than blaKPC) of the CPKP strains, none of the targeted genes was revealed, if excluding the class B (35) blaNDM-negative strains for which blaVIM was not tested. With reference to temporal distribution, from 2012 to 2014 a decrease of blaKPC was observed in contrast to a correspondent increase of blaVIM, which started gradually decreasing from 2015. When the peak of positive rectal swabs (204) was observed in 2015, blaKPC reached the maximum peak frequency (173), doubling that of 2014 and subsequently slightly decreased with a fluctuating trend (figure 2).

When the 22939 patients submitted to CRE surveillance (group 1) were combined with the 1094 CPKPinfected patients (group 2), a total of 1662 CPKP-positive patients was found: 568 CPKP carriers (accounting for $49.4 \%$ of the 1150 patients with CPKP-positive rectal swab), 582 CPKP-infected patients with a CPKP-positive rectal swab (accounting for $50.6 \%$ of the 1150 patients with CPKP-positive rectal swab), 137 CPKP-infected patients with a CPKP-negative rectal swab, and 375 CPKP 


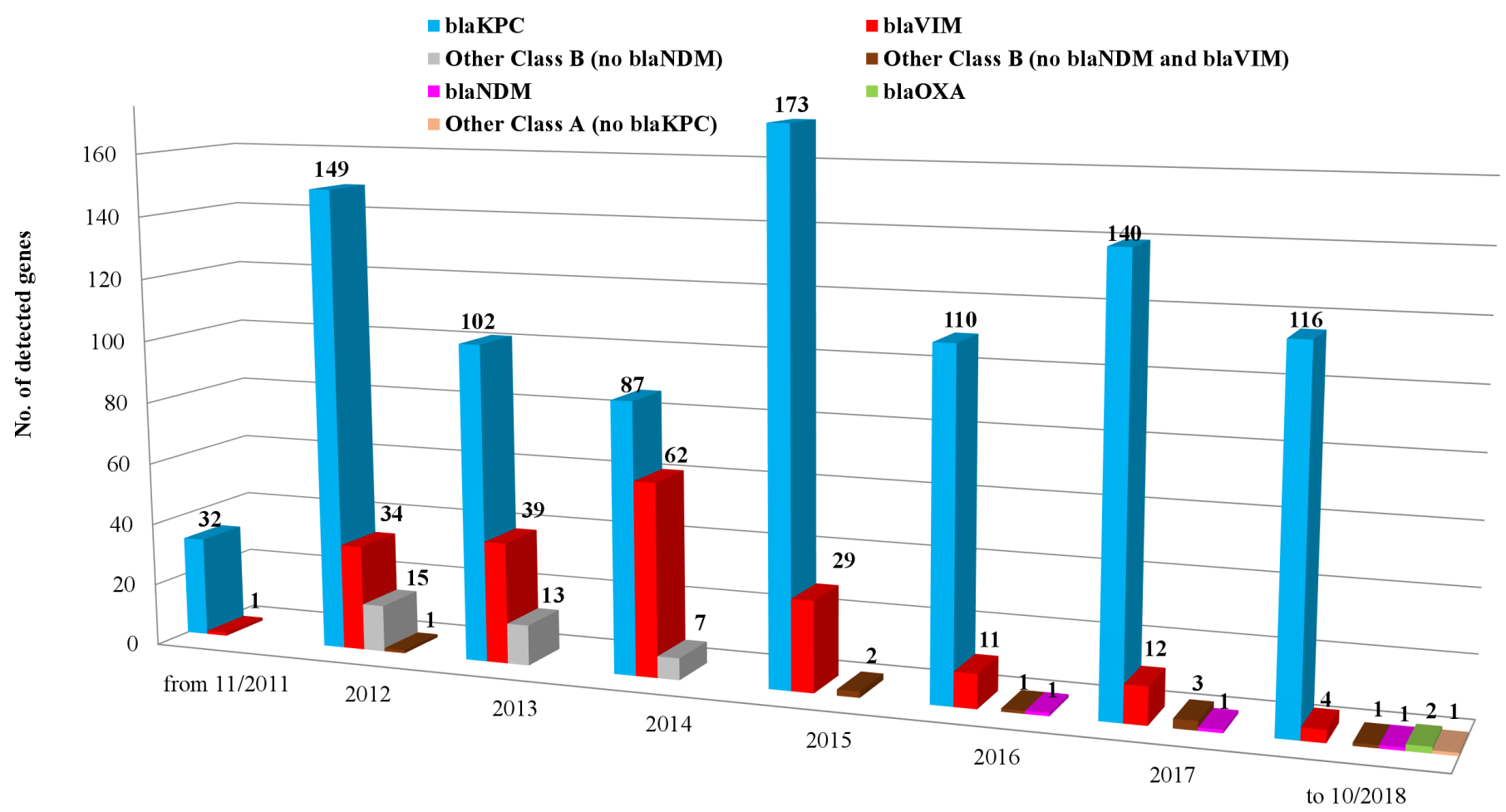

Year

Figure 2 Year distribution of carbapenemase-producing Klebsiella pneumoniae (CPKP) genes in 1150 patients with CPKPpositive rectal swab. KPC, K. pneumoniae carbapenamase; NDM, New Delhi metallo-beta-lactamase; VIM, Verona integronencoded metallo-beta-lactamase; OXA, carbapenem-hydrolyzing oxacillinase.

infected patients not included in the active CRE surveillance (figure 3).

Among the 1094 CPKP-infected patients (719 included in the active CRE surveillance and 375 not included in the active CRE surveillance), accounting for 1283 CPKPpositive samples, urine (675) was the mostly involved sample all over the period, although a significant decrease from 2015 to 2016 was observed. Blood (175) accounted for about 25 cases per year, with a peak in 2017 (34) (figure 4).

With regard to the CPKP-infected patients included in the active CRE surveillance, no significant difference was observed in the frequency of CPKP-positive rectal swabs in the different material groups. On the contrary, CPKPpositive blood (49\%) and respiratory (31\%) samples were more frequently associated than urine $(17.7 \%)$ with CPKP-positive samples from two or more sites of infection $(\mathrm{p}<0.0001)$, as well as CPKP-positive blood samples were more frequently associated with other CPKP-positive samples from two-site or more-site infection $(\mathrm{p}<0.001)$ than the respiratory ones (figure 5).

With reference to the 1094 CPKP-infected patients, $1034(94.5 \%)$ were positive for one of the targeted carbapenemase genes (841 blaKPC, 188 blaVIM, 3 blaOXA48 , and 2 bla NDM) and $5(0.5 \%)$ contained two of the targeted carbapenemase genes $(4$ blaKPC + blaVIMand 1 bla $\mathrm{NDM}+$ blaOXA-48), for a total of 1044 carbapenemase genes detected (figure 6A). If excluding the 50 class B blaNDM-negative strains for which blaVIM was not tested, the remaining 5 cases were negative for the targeted carbapenemase genes (2 class A blaKPC-negative and 3 class B blaVIM and blaNDM negative). When the carbapenemase gene analysis was performed by material grouping, blaKPC was the most frequently detected carbapenemase gene in all material groups, followed by blaVIM; however, the ratio of blaKPC and blaVIM was found to range from 6:1 to 10:1 for all material groups, except for urine for which blaVIM was more significantly detected (ratio 3:1) $(\mathrm{p}<0.01)$ (figure 6B).

\section{DISCUSSION}

Since 2013, the CDC assigned the highest threat level to CRE and declared that CRE require urgent public health attention. ${ }^{89}$ Unlike previous Italian studies reporting the colonisation rate for selected patient categories, ${ }^{10-13}$ our data show the picture of the circulation of CPKP isolates in a single tertiary-care hospital on a big sample size over a long period.

In our experience, the results of the application of active CRE surveillance with the adoption of a combination of phenotypic assays followed by genotypic characterisation on 'at-risk' patient categories highlight the need not to lower the guard about this problem. In fact during the study period, after an initial constant trend of the frequency of CPKP-positive rectal swab cases from 2012 to 2014 (ranging from $4.4 \%$ to $4.7 \%$ ), in 2015 the highest peak $(6.3 \%)$ was observed, in agreement with the same pattern described for invasive infection at regional level, ${ }^{6}$ followed by a decrease in 


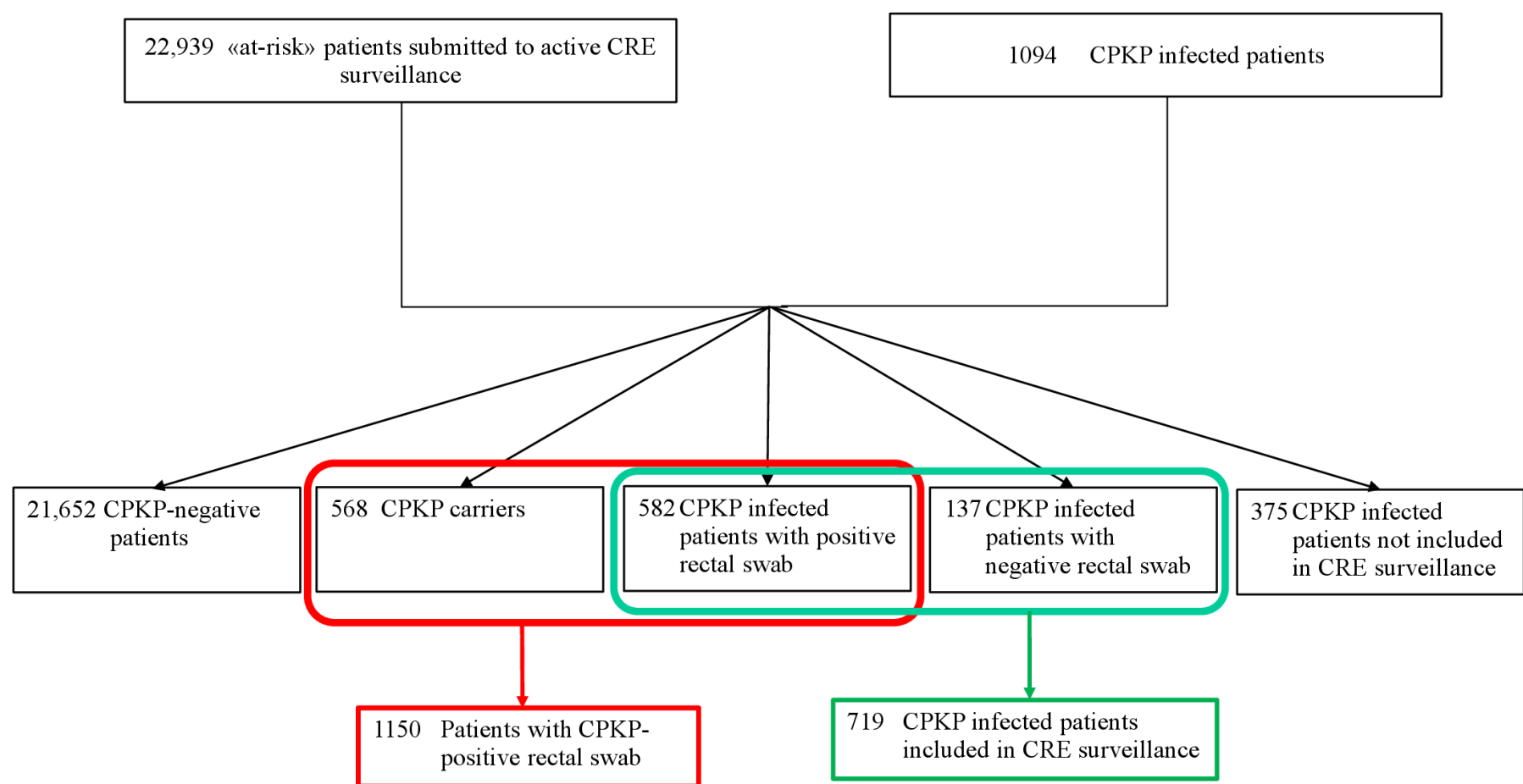

Figure 3 Study result flow diagram. CPKP, carbapenemase-producing Klebsiella pneumoniae; CRE, carbapenem-resistant Enterobacteriaceae.

140

Blood $(n=175) \quad$ Respiratory $(n=221) \quad$ Urine $(n=675)$

Other site $(\mathrm{n}=\mathbf{2 1 2})$

120

106

114

117

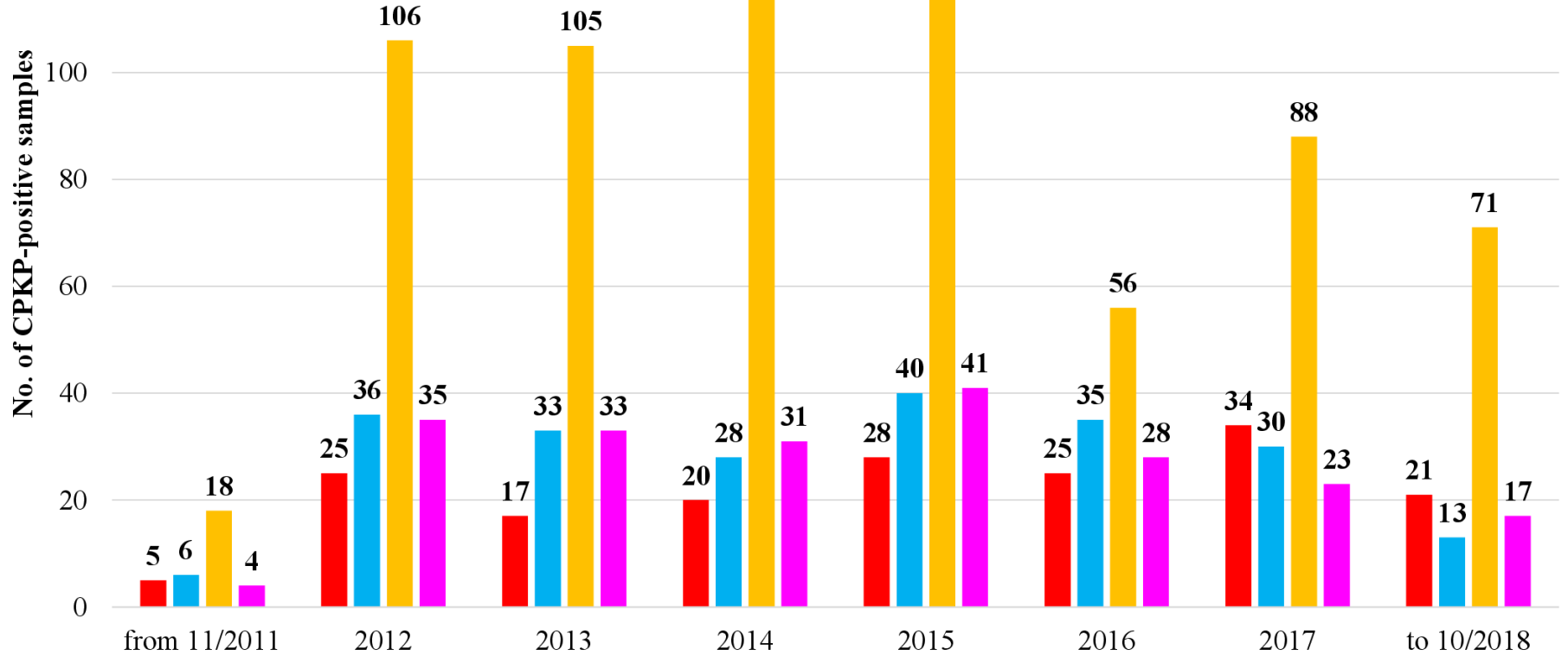

Year

Figure 4 Year distribution of carbapenemase-producing Klebsiella pneumoniae (CPKP)-infected patients by material grouping (blood, respiratory, urine and other sites). 


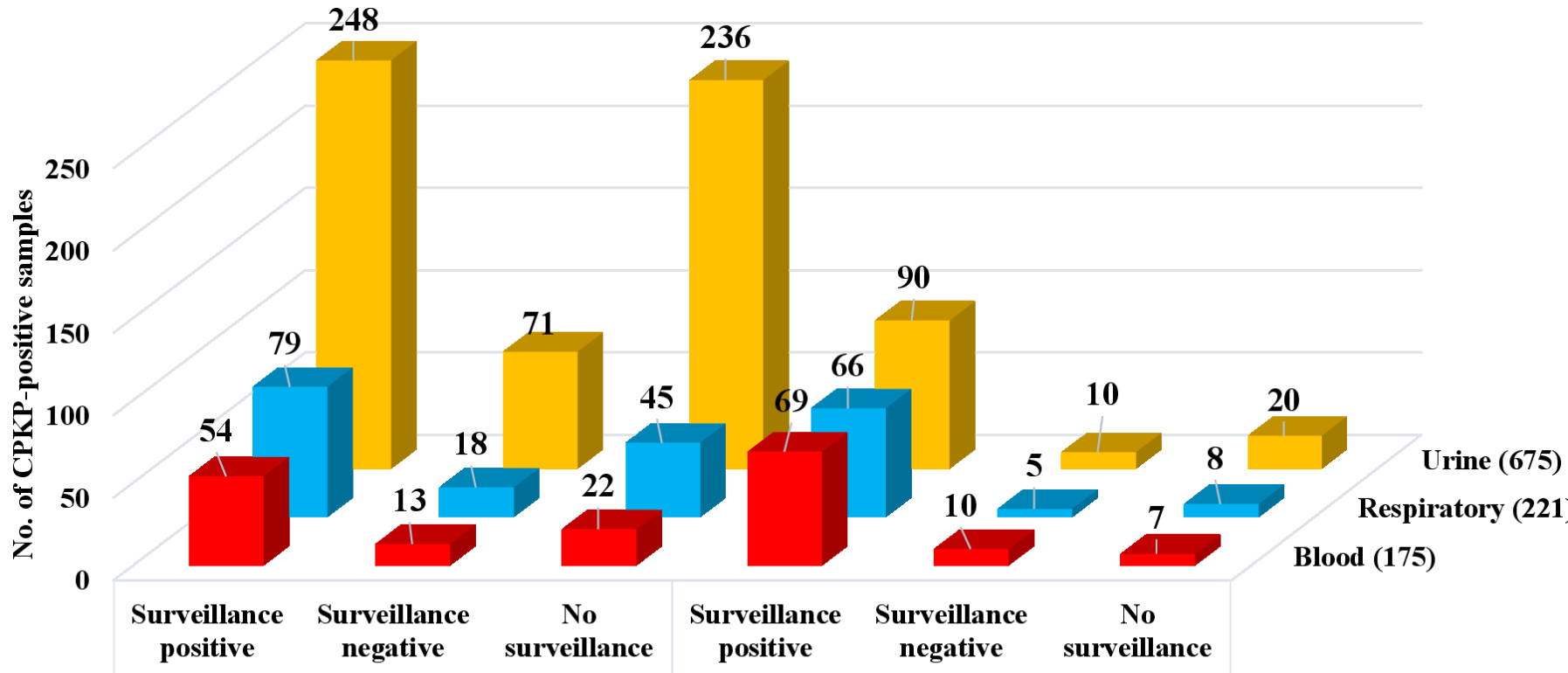

Patients with infection in one-site

Patients with infection in 2 or more sites

Figure 5 Comparison of the results of active carbapenemase-producing Enterobacteriaceae surveillance among carbapenemase-producing Klebsiella pneumoniae (CPKP)-infected patients with one-site infection and in those with multiplesite infection by material grouping (blood, respiratory, urine).

$2016(4.9 \%)$ and a subsequent increasing trend in the last 2 years. The highest prevalence of CPKP-positive rectal swabs was observed in the long-term care units, if excluding those units in which rectal swab screening was performed only on targeted patients, such as contacts of carrier patients and/or transfer from 'atrisk' care units, and obtained on a limited number of rectal swabs.

When the association between patients with CPKPpositive rectal swab and those with CPKP infection

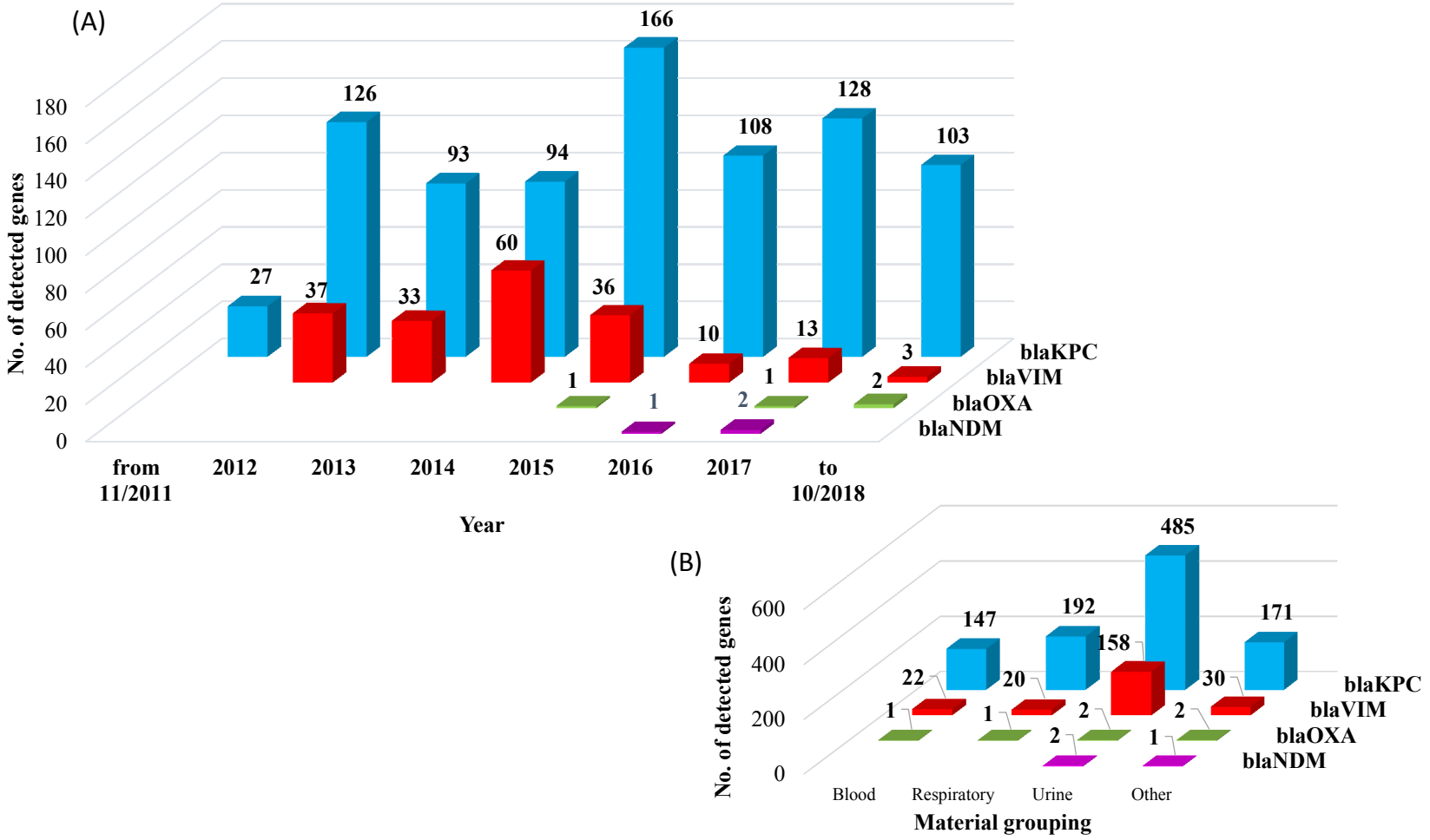

Figure 6 Distribution of carbapenemase-producing Klebsiella pneumoniae (CPKP) genes in infected patients by year (A) and by material grouping (blood, respiratory, urine and other sites) (B). KPC, K. pneumoniae carbapenamase; NDM, New Delhi metallo-beta-lactamase; VIM, Verona integron-encoded metallo-beta-lactamase; OXA, carbapenem-hydrolyzing oxacillinase. 
(CPKP-positive samples other than rectal swab) was considered, it was observed that the $49.4 \%$ of patients submitted to active surveillance with CPKP-positive rectal swab were carriers, representing a potential reservoir for spread of CPKP strains detectable only by surveillance. Taking into account the overall infected patients and excluding those not submitted to active CRE surveillance, no difference was observed in the frequency of patients with CPKP-positive rectal swab among the different material groups. On the contrary, CPKP-positive blood and respiratory samples were more frequently associated with infections in multiple body sites, as expected due to the difficulty in containing invasive infections (blood and respiratory samples) in a unique site and suggesting that carriage represents one of the most important risk factors for CPKP infection, as previously described for bloodstream infection. ${ }^{1415}$

With regard to the temporal distribution of the carbapenemase genes among CPKP-positive rectal swabs, from 2012 to 2014 the blaKPC and blaVIM showed an inverse trend: in fact, when the blaKPC decreased from $74.9 \%$ to $55.8 \%$, the blaVIM increased consequently from $17 \%$ to 39.7\%. In correspondence of the highest CPKP-positive rectal swab rate in 2015, the trend of the frequency of the blaKPC and blaVIM genes has reversed: that of blaKPC has continuously raised again, reaching $92.8 \%$, whereas that of blaVIM progressively decreased to $3.2 \%$. blaNDM and blaOXA-48 were only occasionally detected starting from 2016 and 2018, respectively. A similar temporal distribution was also observed among infected patients, in which blaKPC was prevalent during all the study period with a peak in 2015 and blaVIM was the second most frequently detected carbapenemase gene independently of the material grouping. However, blaVIM was more significantly detected from urine than from other samples. As already described, ${ }^{16} 17$ in Italy blaKPC remains endemic and blaVIM is the predominant MBL whereas the blaNDM is only sporadically detected, in agreement with the findings in our single-centre study. However, our data are in contrast with those recently reported to the Italian national surveillance from 2014 to 2017 in bloodstream infections, for which the emergence of blaOXA48, especially in CPKP isolates, and its assessment as the first most common gene after blaKPC, overcoming blaVIM, were described. ${ }^{18}$ The overall high prevalence of blaVIM, mainly due to the relative peaks observed in 2013 and 2014 in our study, could be explained by a possible outbreak of the same clone of blaVIM among patients attending long-term care wards.

There are a few limitations in this study. First, this is a single-centre study and the findings may not generalise well to other settings due to multiple local factors. However, the carbapenemases have a global distribution, but substantial over time variability can be observed not only at continental and national level, but also among different settings in the same region: global data derive from single-centre studies. Awareness on the distribution of the specific mechanisms of carbapenem resistance within Enterobacteriaceae, in particular K. pneumoniae, and their temporal trend is crucial in the prevention of their spread and selection of appropriate patient management. Second, the genotypic characterisation was limited to the detection of the four major carbapenemase genes that could have missed the more rarely circulating genotypes. Third, the lack of further genetic typing hampered to add consideration about any molecular epidemiological link among the isolates. Nonetheless, these data emphasise the importance of active surveillance for timely detection and separation of carriers, activation of contact precautions and, after risk evaluation, antibiotic treatment guidance on suspicion of infection, besides the evaluation of the risk factors for invasive infections, avoiding unnecessary potential toxic antimicrobial therapy in low-risk patients and for starting adequate treatment promptly in those at high risk.

Contributors $A C, M M$ and $C C$ conceived and designed the study. AC, MB, MM, SM, SC, AR, IR, ADM, MG and SL acquired the data. AC, MB, MM, SM, MCA and FDC analysed and interpreted the data. AC, MB, MM, SM and CC drafted the article or revised it critically. AC, MB, MM, SM, SC, AR, IR, ADM, MG, SL, MCA, CC and FDC finally approved the version to be submitted.

Funding This study was supported by the Ministry of University and Scientific Research Grant FIL, University of Parma, Italy. Grant number: N/A.

Competing interests None declared.

Patient and public involvement Patients and/or the public were not involved in the design, or conduct, or reporting, or dissemination plans of this research.

Patient consent for publication Not required.

Provenance and peer review Not commissioned; externally peer reviewed.

Data availability statement All data relevant to the study are included in the article or uploaded as supplementary information.

Open access This is an open access article distributed in accordance with the Creative Commons Attribution Non Commercial (CC BY-NC 4.0) license, which permits others to distribute, remix, adapt, build upon this work non-commercially, and license their derivative works on different terms, provided the original work is properly cited, appropriate credit is given, any changes made indicated, and the use is non-commercial. See: http://creativecommons.org/licenses/by-nc/4.0/.

ORCID iD

Adriana Calderaro http://orcid.org/0000-0002-4308-3120

\section{REFERENCES}

1 Seekatz AM, Bassis CM, Fogg L, et al. Gut microbiota and clinical features distinguish colonization with Klebsiella pneumoniae carbapenemase-producing Klebsiella pneumoniae at the time of admission to a long-term acute care hospital. Open Forum Infect Dis 2018;5:ofy190.

2 Falagas ME, Tansarli GS, Karageorgopoulos DE. Deaths attributable to Enterobacteriaceae infections. Emerg Infect Dis 2014;20:1170-5.

3 Calderaro A, Buttrini M, Piergianni M, et al. Evaluation of a modified meropenem hydrolysis assay on a large cohort of KPC and VIM carbapenemase-producing Enterobacteriaceae. PLoS One 2017;12:e0174908-12.

4 Bonomo RA, Burd EM, Conly J, et al. Carbapenemase-producing organisms: a global scourge. Clin Infect Dis 2018;66:1290-7.

5 Queenan AM, Bush K. Carbapenemases: the versatile betalactamases. Clin Microbiol Rev 2007;20:440-58.

6 Agenzia Sanitaria Regione Emilia-Romagna. Indicazioni pratiche E protocolli operativi per La diagnosi, La sorveglianza E IL controllo degli enterobatteri produttori di carbapenemasi nelle strutture sanitarie E socio-sanitarie, 2017. Available: http://assr.regione.emiliaromagna.it/it/servizi/pubblicazioni/rapporti-documenti/indicazionipratiche-diagnosi-cpe-2017 
7 Horan TC, Andrus M, Dudeck MA. CDC/NHSN surveillance definition of health care-associated infection and criteria for specific types of infections in the acute care setting. Am J Infect Control 2008;36:309-32.

8 Centers for Disease Control and Prevention. Antibiotic resistance threats in the United States, 2013, 2013. Available: http://www.cdc. gov/drugresistance/pdf/ar-threats-2013-508.pdf

9 World Health Organization. Guidelines for the prevention and control of carbapenem-resistant Enterobacteriaceae, Acinetobacter baumannii and Pseudomonas aeruginosa in health care facilities, 2017. Available: www.who.int/infection-prevention/en

10 Nucleo E, Caltagirone M, Marchetti VM, et al. Colonization of longterm care facility residents in three Italian provinces by multidrugresistant bacteria. Antimicrob Resist Infect Control 2018;7:33.

11 Giannella M, Bartoletti M, Morelli MC, et al. Risk factors for infection with carbapenem-resistant Klebsiella pneumoniae after liver transplantation: the importance of pre- and posttransplant colonization. Am J Transplant 2015;15:1708-15.

12 Tedeschi S, Trapani F, Liverani A, et al. The burden of colonization and infection by carbapenemase-producing Enterobacteriaceae in the neuro-rehabilitation setting: a prospective six-year experience. Infect Control Hosp Epidemiol 2019;40:368-71.

13 Castagnola E, Tatarelli P, Mesini A, et al. Epidemiology of carbapenemase-producing Enterobacteriaceae in a pediatric hospital in a country with high endemicity. $J$ Infect Public Health 2019;12:270-4.

14 Giannella M, Trecarichi EM, De Rosa FG, et al. Risk factors for carbapenem-resistant Klebsiella pneumoniae bloodstream infection among rectal carriers: a prospective observational multicentre study. Clin Microbiol Infect 2014;20:1357-62.

15 Bassetti M, Giacobbe DR, Giamarellou H, et al. Management of KPCproducing Klebsiella pneumoniae infections. Clin Microbiol Infect 2018;24:133-44.

16 Albiger B, Glasner C, Struelens MJ, et al. Carbapenemase-producing enterobacteriaceae in Europe: assessment by National experts from 38 countries, may 2015. Euro Surveill 2015;20.

17 van Duin D, Doi Y. The global epidemiology of carbapenemaseproducing Enterobacteriaceae. Virulence 2017;8:460-9.

18 lacchini S, Sabbatucci M, Gagliotti C, et al. Bloodstream infections due to carbapenemase-producing Enterobacteriaceae in Italy: results from nationwide surveillance, 2014 to 2017. Euro Surveill 2019;24. 